\title{
Acesso de trabalhadores de um território aos serviços ofertados pela unidade básica de saúde
}

\author{
Access by workers of a territory to the services offered by the primary care unit
}

Acceso de los trabajadores de un territorio a los servicios ofrecidos por la unidad de atención primaria

\author{
Christiane Gleyce da Silva' @; Rafaella Almeida Fortes' @ - Cristiane Helena Gallasch' @ ; \\ Luciana Valadão Vasconcelos Alves" 르; Delson da Silval 르; Magda Guimarães de Araujo Farial 르 \\ 'Universidade do Estado do Rio de Janeiro, Rio de Janeiro, RJ, Brasil; "Instituto Federal Fluminense, Macaé, RJ, Brasil
}

\begin{abstract}
RESUMO
Objetivo: analisar as condições do acesso de trabalhadores terceirizados às atividades de saúde realizadas em uma Unidade Básica de Saúde em seu território de abrangência. Método: estudo de natureza descritiva e qualitativa, com coleta de dados realizada por meio de entrevistas semi-estruturadas com 15 trabalhadores, no segundo semestre de 2018, após autorização do comitê de ética em pesquisa. A análise foi realizada a partir do referencial teórico-conceitual da análise de conteúdo temáticocategorial. Resultados: após análise, emergiram duas categorias temáticas, a citar: Entraves na absorção de trabalhadores do território nas unidades básicas de saúde e Estratégias para a busca de serviços de saúde: serviços particulares e emergências como porta de entrada. Conclusão: percebeu-se que os participantes enfrentam grandes desafios para alcançar a porta de entrada do SUS, sobretudo, devido a inabilidade dos serviços de saúde no atendimento a indivíduos trabalhadores inseridos no território de responsabilidade sanitária.
\end{abstract}

Descritores: Atenção Primária à Saúde; Acesso aos Serviços de Saúde; Saúde do Trabalhador; Promoção da Saúde.

\section{ABSTRACT}

Objective: to examine the conditions of outsourced workers' access to health activities carried out in the catchment territory of a primary care facility. Method: in this qualitative, descriptive study, after research ethics committee approval, data were collected in the second half of 2018 by semi-structured interviews of 15 workers, and analyzed within the theoretical-conceptual framework of thematic-category content analysis. Results: after analysis, two thematic categories emerged: barriers to health units' absorbing workers from the territory; and strategies for seeking out health services: private and emergency services as gateways. Conclusion: the participants were found to face major challenges in reaching the public health service (SUS) gateway, particularly due to health services' inability to provide care to individual workers in the health territory under their responsibility.

Descriptors: Primary Health Care; Health Services Accessibility; Occupational Health; Health Promotion.

\section{RESUMEN}

Objetivo: analisar las condiciones de acceso de los trabajadores subcontratados a las actividades de salud realizadas en el territorio de captación de uma unidad de atención primaria. Método: en este estudio cualitativo, descriptivo, luego de la aprobación del comité de ética de la investigación, se recolectaron datos en el segundo semestre de 2018 mediante entrevistas semiestructuradas a 15 trabajadores, y se analizaron dentro del marco teórico-conceptual del análisis de contenido de categorías temáticas. Resultados: luego del análisis, surgieron dos categorías temáticas: barreras para que las unidades de salud absorban trabajadores del territorio; y estrategias de búsqueda de servicios de salud: servicios privados y de emergencia como pasarelas. Conclusión: se encontró que los participantes enfrentan grandes desafíos para llegar a la puerta de entrada del servicio público de salud (SUS), particularmente debido a la incapacidad de los servicios de salud para brindar atención a los trabajadores individuales en el territorio de la salud bajo su responsabilidad..

Descriptores: Atención Primaria de Salud; Accesibilidad a los Servicios de Salud; Salud Laboral; Promoción de la Salud.

\section{INTRODUÇÃO}

A Atenção Primária à Saúde (APS) tem como objetivo promover um conjunto de ações no âmbito da saúde, de maneira individual e coletiva, com o intuito de garantir a promoção da saúde, a prevenção de agravos, o diagnóstico, o tratamento, a reabilitação, a redução de danos e a manutenção da saúde, com a finalidade de desenvolver uma atenção integral que intervém na situação de saúde e autonomia das pessoas e nos determinantes e condicionantes de saúde das coletividades ${ }^{1}$.

Ao se analisar o acesso de trabalhadores a esse serviço, constata-se uma baixa adesão, impedindo que ações de prevenção de doenças e promoção da saúde possam ser efetivas. Assim, para garantir o acesso do trabalhador às ações de saúde realizadas no âmbito da APS, criou-se a Política Nacional de Saúde do Trabalhador e da Trabalhadora ${ }^{2}$, que considera todas as necessidades de segurança e direito à saúde do trabalhador e por meio de um cuidado integral, faz

Autora Correspondente: Magda Guimarães de Araújo Faria. E-mail: magda.faria@live.com.

Editor responsável: Octavio Muniz da Costa Vargens. 
com que as ações de vigilância se tornam cada vez mais efetivas e reduza morbimortalidade relacionada a esse ambiente. Ela também enfatiza o direito à saúde de todo trabalhador brasileiro, conforme previsto na Constituição Federal $^{3}$, indicando que, urbano ou rural, empregado ou desempregado, o trabalhador, homem ou mulher, tem direito ao acesso e aos cuidados do serviço de saúde.

Dito isto, percebe-se a necessidade de realizar ações que possam promover a integralidade e a vigilância à saúde do trabalhador (VISAT), a citar: a identificação da rotina de trabalho; a análise dos riscos e vulnerabilidades que o trabalho impõe; o levantamento das demandas de saúde do território e; a análise do ambiente, intervindo quando necessário ${ }^{4}$.

Apesar de serem ações tangíveis e com grande impacto à saúde do trabalhador, a prática da VISAT ainda é permeada de fatores dificultadores, como por exemplo, o próprio mundo do trabalho que, devido a longas jornadas e distanciamento do local de moradia, não permitem que o trabalhador seja acompanhado por sua unidade básica de saúde (UBS) de referência 5 .

É válido ressaltar que as dificuldades são ainda mais intensas quando se trata de trabalhadores terceirizados, já que estes, historicamente, possuem contratos de trabalho formados por longas jornadas, baixos vencimentos e elevados índices de adoecimentos. Observa-se esta fragilidade em todos os setores, sobretudo, nos serviços de vigilância e asseio e conservação ${ }^{6}$.

Neste sentido, propõe-se como objetivo geral analisar as condições do acesso de trabalhadores terceirizados às atividades de saúde realizadas em uma Unidade Básica de Saúde em seu território de abrangência.

Acredita-se que o presente estudo possa contribuir na melhoria para o acesso dos trabalhadores aos serviços da APS, assim como discutir estratégias específicas para esse público, aperfeiçoando o conhecimento dos profissionais e acadêmicos, proporcionando a base necessária para a contínua evolução do Sistema Único de Saúde e aplicação de seus princípios, dando possibilidade a todo trabalhador e trabalhadora acessar os serviços de saúde.

\section{MÉTOdo}

Pesquisa qualitativa, descritiva e não-experimental. A coleta de dados foi realizada junto a trabalhadores terceirizados atuantes em uma instituição de ensino superior no estado do Rio de Janeiro. A população total foi de 35 trabalhadores, entretanto apenas 15 optaram por participar da pesquisa. Ressalta-se que não houve técnica de amostragem. A captação de participantes se deu a partir de uma amostra não probabilística que utiliza cadeias de referência, comumente chamada de "bola de neve" ${ }^{\text {" }}$.

A técnica para a coleta de dados foi a entrevista semi-estruturada, composta por dez questões. As entrevistas foram gravadas em gravador MP3, e posteriormente transcritas. A análise das entrevistas deu-se à luz do método da análise de conteúdo temático-categorial, cujas etapas são: 1. Pré-análise, 2. Exploração do material; 3. Tratamento dos resultados ${ }^{8}$.

Esta pesquisa foi submetida à Plataforma Brasil, onde posteriormente foi avaliada por um Comitê de Ética em Pesquisa, respeitando todos os critérios éticos conforme dispositivos legais em vigor no território nacional ${ }^{9}$. Neste sentido, ressalta-se que os dados foram coletados e analisados no segundo semestre de 2018, após aprovação do projeto sob o número de parecer 2.582.140.

\section{RESULTADOS}

Observou-se que dentre a população participante do estudo, $40 \%$ era composta de auxiliares de serviços gerais e conservação, $40 \%$ trabalhavam na segurança patrimonial, $13 \%$ atuavam como ascensorista de elevadores e $7 \%$ faziam parte do corpo técnico-administrativo. A faixa etária mais prevalente foi entre 35 e 44 anos, na qual encontram-se $47 \%$ dos trabalhadores. Além disso, $67 \%$ dos entrevistados eram do sexo feminino e, apenas $40 \%$ possuíam o mais alto grau de escolaridade encontrado na pesquisa, ou seja, ensino médio completo. Aproximadamente $60 \%$ dos entrevistados afirmaram não possuir folgas semanais devido a outras tarefas remuneradas durante as folgas na instituição de coleta.

Observou-se que os trabalhadores possuem turnos e jornadas de trabalho diferentes, conforme a função exercida. Identificou-se que os profissionais da segurança possuem escalas de doze por trinta e seis horas, o que permite maior flexibilidade de horários para realizar atividades, desde consultas ou atividades de lazer, como também acrescentar uma segunda jornada de trabalho. As ascensoristas, trabalham seis horas por dia em turnos diferentes e, em virtude disso, quando uma funcionária necessita faltar por algum motivo, a profissional que está em serviço deve permanecer na segunda jornada de trabalho. Os profissionais do serviço de conservação e limpeza e de secretariado são os mais afetados, pois trabalham oito horas por dia, incluindo finais de semana e, portanto, encontram mais dificuldades para realizar algum acompanhamento de saúde.

Sobre a existência de UBS próxima ao trabalho, $87 \%$ indicaram ter conhecimento da UBS localizada a poucos metros da instituição, entretanto, destes, apenas $20 \%$ referiram frequentar uma unidade de cuidados primários de saúde, sendo 
todas as respostas voltadas para a unidade de referência próxima à sua residência. Do total de entrevistados, $45 \%$ referiram preferir utilizar a rede privada de saúde, devido, essencialmente, a dificuldade de acesso na rede pública.

A partir dos discursos dos profissionais a análise emergiu para a construção de duas categorias temáticas, a citar: Entraves na absorção de trabalhadores do território nas unidades básicas de saúde e, Estratégias para a busca de serviços de saúde: serviços particulares e emergências como porta de entrada.

\section{Entraves na absorção de trabalhadores do território nas unidades básicas de saúde}

Este tema trata sobre a dificuldade de estabelecer os princípios legais de oferta de serviços para todos os trabalhadores do território nacional. Assim sendo, os trabalhadores relatam já terem tido o interesse em se cadastrar na UBS próxima ao trabalho, porém não foram cadastrados pelo fato de não residirem no território:

A exigência deles de comprovante de residência, porque você não mora aqui e trabalha aqui, é burocracia para eles [...] eu moro em outra cidade e trabalho aqui, mas se eu estou trabalhando aqui, se eu conseguisse atendimento aqui seria muito mais fácil, evitaria de faltar trabalho, eu poderia simplesmente sair do meu trabalho e ser atendido na clínica (E4).

Observa-se também que segundo os participantes, as diferenças políticas na estrutura e oferta da APS interferem no atendimento:

Onde eu moro é um pouco difícil, porque lá onde eu moro não é clínica da família é postinho, mas aqui onde eu trabalho é mais perto, mas eles não atendem a gente (E12).

Neste sentido, as ações da UBS no território de atuação laboral são restritas basicamente a campanhas de vacinação:

Ali eu só vou pra tomar vacina (E11).

Nessa aqui não tem nada pra gente que trabalha e perto da minha casa eu só vou pra pegar remédio. Clínica da família é só pra isso, pra pegar os medicamentos (E6).

Outra questão que também deve ser observada é a inflexibilidade do trabalho para o acompanhamento da saúde do trabalhador. Assim sendo, existe um déficit na oferta de atividades de promoção da saúde e prevenção de agravos. Isso está relacionado a insegurança no ambiente laboral e do receio que as faltas se tornem um problema na avaliação do desempenho do trabalhador.

Só quando passo mal, periódico não (E2).

Eu não tenho tempo né, por causa do trabalho que não libera, só em caso de emergência (E11).

A precarização dos serviços de saúde é outro ponto importante a ser mencionado como desestimulador desse acesso, uma vez que, tendo a possibilidade de ir a uma UBS, muitas vezes o trabalhador não consegue ser atendido devido a falta de profissionais, a grande demanda ou a ausência de seguimento no acompanhamento.

Me marcaram mamografia umas três ou quatro vezes, para onde me mandaram os tomógrafos estavam quebrados e até hoje não consegui fazer (E6).

\section{Estratégias para a busca de serviços de saúde: serviços particulares e emergências como porta de entrada}

A dificuldade de acesso aos serviços da APS repercute na busca por estratégias alternativas para o atendimento em saúde. Neste sentido, observou-se o estabelecimento de duas principais fontes de serviços de saúde: os serviços particulares e as emergências. Esta categoria irá discutir seus impactos sob o prisma dos trabalhadores.

A dificuldade no acesso aos serviços da APS afasta o trabalhador do que seria sua principal porta de entrada ao Sistema Único de Saúde (SUS), aproximando-o e incentivando-o a buscar a rede particular, que é indicada pelos participantes como mais flexível e resolutiva:

Eu já tive plano de saúde da outra empresa que eu trabalhei, mas no momento tive que cancelar que a situação [...] foi ficando muito cara. Se der mais tarde para voltar, a gente faz um plano familiar de novo (E15).

Se a gente for olhar assim, o particular é bem melhor, mas a gente não pode, eu não posso pagar um plano, aí eu tenho que fica na clínica da família, que eu não tenho tempo de ir e depender do SISREG que fica a vida toda (E13).

É importante salientar que os danos causados a saúde do trabalhador agravam-se, diante da ausência no acompanhamento eficaz, pois o indivíduo encontra-se em situação de vulnerabilidade, definido através dos inúmeros riscos laborais existentes, doenças pregressas e atuais, fatores de riscos externos ao trabalho, ausência dos serviços de VISAT e a limitação da integração dos serviços de saúde.

Sendo assim, o ideal seria o estabelecimento de atividades baseadas na promoção de saúde, entretanto não é esta a realidade: 
Tem mais de três anos que eu não vou no médico, só quando to morrendo, passando mal, aí tem que ir, não tem jeito (E4).

Pode-se inferir que o trabalhador não consegue ter acesso aos serviços da UBS, delimitando que o cuidado a ser exercido sobre ele, será realizado somente em último caso, diante do agravo de doenças:

Mais fácil pagar uma emergência e mesmo assim demora um pouquinho, mas a gente é tratada melhor que no público (E13).

A priorização pelos atendimentos em emergências, como parte central do cuidado a saúde interfere diretamente na rotina de trabalho, impedindo muitas vezes que o trabalhador continue exercendo determinadas atividades no local, afetando todo o sistema de trabalho:

O problema com o meu pé, eu já fiquei sem conseguir colocar o pé no chão, aí não tinha como eu vir, a sorte é que o pessoal aqui sabe que eu tenho esse problema e quando eu realmente não tenho como trabalhar, eu não venho (E6).

Observa-se que os trabalhadores possuem a percepção de que este não é o meio ideal para se adentrar ao serviço de saúde, mas a incompatibilidade temporal aponta esta como a única alternativa:

Como eu trabalho direto, geralmente eu esqueço, não acho tempo para ir no médico, só lembro quando to morrendo mesmo, ai eu vou para emergência (E4).

Eu acho que é melhor ir na clínica da família do que ir na emergência, porque as vezes você vai aí não resolve e lá na clínica, eles atendem e, se tiver alguma coisa grave eles já te encaminham para um hospital. $O$ problema é conseguir atendimento (E10).

\section{DISCUSSÃO}

A APS é responsável pela atenção à saúde dos usuários, por meio de ações de saúde de caráter individual e coletivo e, apesar de contribuir para melhor a acessibilidade dos indivíduos, observa-se a desproporção entre oferta, capacidade de atendimento e demanda. Além disso, fatores como acesso, marcação de consulta, excesso de demanda e tempo de espera, nível de satisfação do usuário, vínculo, ausência de articulação em redes integradas e as ações baseadas no modelo biomédico hegemônico são relatados como limitadores para o bom funcionamento e o uso da rede $\mathrm{e}^{10-11}$.

A integralidade defende o direito do usuário acessar os serviços de saúde em todos os níveis de atenção, além de referir-se à articulação entre a prevenção das doenças, promoção da saúde e assistência, considerando às demandas objetivas e subjetivas das pessoas em seu contexto social ${ }^{12-13}$. Ademais, a ESF, além de possibilitar o acesso universal e contínuo a serviços de saúde de qualidade e resolutivos, deve realizar o acolhimento dos usuários. Parte do princípio de que a UBS deve receber e ouvir todos os que procuram seus serviços ${ }^{1,14}$. No entanto, os princípios da integralidade, universalidade e acolhimento esbarram no fundamento de que a ESF atue a partir de áreas geográficas definidas, ou seja, cada UBS possui uma população adscrita e não realiza atendimento para além dessa população. No entanto, recorda-se que o território de abrangência da ESF é não apenas "depositário de atributos da população; seria também o lugar da responsabilidade e da atuação compartilhada"15

Dessa forma, observa-se um grande entrave, por um lado, o trabalhador não está acessando a rede como deveria, uma vez que, políticas públicas asseguram o acesso a todos os níveis de serviços de saúde, independente do território onde esse indivíduo resida ${ }^{2}$ e, por outro lado, o desconhecimento do direito ao acesso universal, equânime e integral à saúde prejudica a reivindicação à estes serviços ${ }^{16}$.

Em que pese os direitos à saúde, é necessário ressaltar que a responsabilização da APS, sobretudo da ESF se dá na organização do território sanitário cuja oferta de serviços deve ser compatível com a necessidade dos usuários, no que tange às demandas de ações de promoção da saúde, prevenção de agravos e atividades curativas ${ }^{1}$. Entretanto, ainda são observadas poucas iniciativas para o atendimento de indivíduos fora do território-moradia, ainda que este seja o local aonde estes passam a maior parte do dia. Neste sentido, observa-se que apenas as notificações de agravos relacionados ao trabalho (acidentes de trabalho e doenças ocupacionais) são muitas vezes, as únicas ações voltadas para a saúde de trabalhadores na APS ${ }^{17}$.

Para suprir as necessidades deste déficit de atendimento, os trabalhadores buscam prioritariamente, o atendimento em unidades de emergência. Essa é uma tendência em todo território nacional, entretanto, observa-se que boa parte desses atendimentos são classificados como ambulatoriais, ou seja, poderiam ser resolvidos na própria APS ${ }^{18-19}$.

Em relação à busca pelo setor privado, esta se dá sobretudo pela demora no atendimento no serviço público, seja no tempo de espera ou na resolutividade da demanda. A linha histórica do SUS indica que o número de médicos atuantes na APS quadruplicou nos últimos 30 anos, contudo, este número ainda é incompatível com as necessidades da população ${ }^{20}$. Sendo assim, o SUS vem se consolidando enquanto sistema público que convive com um sólido sistema privado de saúde suplementar e complementar ${ }^{21}$ 
Não obstante a isso, ressalta-se a grande recessão econômica e orçamentária do SUS, sentida nos últimos anos em todos os campos da saúde e, na APS sob ausência de insumos, profissionais e medicamentos, culminando em redução de equipes e aumento na carga de trabalho dos profissionais atuantes ${ }^{22}$.

Neste sentido, é perceptível o crescimento do setor privado na busca pelas ações de saúde, já que estas muitas vezes estão inseridas dentro do contexto público de maneira predatória e quase imperceptível ${ }^{23}$. Além disso, há uma forte tendência popular em associar o serviço público e gratuito de saúde à ações ineficazes geralmente baseadas em situações de sucateamento ou má gestão pública dos recursos ${ }^{24}$.

O acesso à saúde não se restringe apenas a utilização dos serviços, pois, inclui, concomitantemente, o uso de serviços de qualidade, no tempo adequado e que supram as demandas da população de modo resolutivo ${ }^{25}$.

A prevenção de doenças e promoção a saúde quando assumidas no seu real papel, interferem positivamente na realidade dos usuários, visto que impossibilita o agravamento de danos gerados ${ }^{26}$, inclusive quando se utiliza os serviços de notificação, empregados pela VISAT, vigilância epidemiológica, entre outros. Esses serviços de vigilância podem direcionar assertivamente estratégias para acolher e atender esses usuários, compreendendo que o ambiente ocupacional é um fator importante, que irá influenciar na forma como esse trabalhador cuida de si próprio.

Vale ressaltar, que o olhar diferenciado das equipes de saúde, aliado à construção de estratégias que viabilizem a maior assistência no território de cobertura sanitária, ainda se constitui como desafio, o que dificulta a capacidade de respostas para melhorar a prevenção, promoção e notificação, interferindo diretamente no entendimento das rotinas de trabalho. É necessário o desenvolvimento de estratégias e intervenções que gerem mudanças nos fatores prejudiciais ao trabalhador, reduzindo assim, sua vulnerabilidade ${ }^{16}$.

Já em relação a questão do trabalho como dificultador do acesso aos serviços de saúde, percebe-se que os empregadores pouco propõem ações para reverter tais barreiras, como flexibilização de horários, programação de folgas segundo a demanda do trabalhador e implementação de atividades de promoção à saúde no ambiente laboral. É importante que os empregadores compreendam que promover a saúde e prevenir as doenças dos trabalhadores acarreta em bem-estar coletivo, o que repercute positivamente na produtividade e no desenvolvimento da empresa ${ }^{26}$.

\section{CONCLUSÃO}

Observou-se que os participantes enfrentam grandes desafios para alcançar a porta de entrada do SUS, sobretudo, devido a inabilidade dos serviços de saúde no atendimento a indivíduos trabalhadores inseridos no território de responsabilidade sanitária.

Tais dificuldades geram estratégias alternativas de enfrentamento a falta de atendimento na APS. A primeira estratégia é a busca por serviços de saúde particulares, os quais geram repercussões financeiras para o trabalhador. Além disso, como outra possibilidade, o trabalhador lança mão de busca por cuidados de saúde apenas em casos de emergência, culminando em descontinuidades na integralidade da atenção e, agravamento de quadros que poderiam ser controlados com ações de promoção da saúde.

A APS deve gerenciar as redes de atendimento, de forma articulada, considerando as políticas e diretrizes que foram instituídas para alcançar toda população, incluindo os trabalhadores. Neste sentido, é necessária uma transformação do processo de trabalho, direcionando o atendimento ao foco original da ESF baseado na responsabilidade sanitária sobre o território.

\section{REFERÊNCIAS}

1. Ministério da Saúde (BR). Politica Nacional de Atenção Básica. Portaria no 2.436, de 21 de setembro de 2017. Brasília, 2017 [cited 2019 Jul 22]. Available from: https://bvsms.saude.gov.br/bvs/saudelegis/gm/2017/prt2436_22_09_2017.html

2. Ministério da Saúde (BR). Política Nacional de Saúde do Trabalhador e da Trabalhadora. Portaria no 1.823, de 23 de agosto de 2012. Brasília, 2012 [cited 2019 Jul 22]. Available from:

http://bvsms.saude.gov.br/bvs/saudelegis/gm/2012/prt1823_23_08_2012.html

3. Senado Federal (BR). Constituição da República Federativa do Brasil. Texto constitucional promulgado em 5 de outubro de 1988. Brasília, 1988 [cited 2019 Jul 22]. Available from: https://www2.senado.leg.br/bdsf/bitstream/handle/id/518231/CF88_Livro_EC91_2016.pdf

4. Vianna LCR, Ferreira AP, Vasconcellos LCF de, Bonfatti RJ, Oliveira MHB de. Occupational Health Surveillance: a study in the light of Ministerial Directive no 3.120/98. Saúde em Debate [Internet]. 2017 [cited 2020 Jul 27]; 41(114):786-800. DOI: https://doi.org/10.1590/0103-1104201711409

5. Lacaz FA de C, Trapé A, Soares CB, dos Santos APL. Family health strategy and workers' health: is a dialogue possible? Interface comun. saúde educ. [Internet]. 2013 [cited 2020 Jul 27]; 17(44):75-87. DOI: http://dx.doi.org/10.1590/S1414-

32832013000100007 
6. Pelatieri P, Marcolino A, Horie L, Costa LAR da, Camargos RC. As desigualdades entre trabalhadores terceirizados e diretamente contratados: Análise a partir dos resultados de negociações coletivas de categorias selecionadas. In: Ipea, editor. Terceirização do Trabalho no Brasil: novas e distintas perspectivas para o debate [Internet]. Brasília, 2018 [cited 2020 Jul 27]; p. 33-48. Available from: http://repositorio.ipea.gov.br/bitstream/11058/8702/1/As\%20Desigualdades.pdf

7. Vinuto J. A amostragem em bola de neve na pesquisa qualitativa: um debate em aberto. Temáticas [Internet]. 2016 [cited 2019 Jul 22]; 22(44):2013-220. Available from: https://www.ifch.unicamp.br/ojs/index.php/tematicas/article/view/2144/1637

8. Oliveira DC. Theme/category-based content analysis: a proposal for systematization. Rev. enferm. UERJ [Internet]. 2008 [cited 2019 Jul 22]; 16(4):569-76. Available from: http://www.revenf.bvs.br/pdf/reuerj/v16n4/v16n4a19.pdf

9. Ministério da Saúde (BR), Conselho Nacional de Saúde. Resolução No 466 de 16 de dezembro de 2012. Diretrizes e normas regulamentadoras de pesquisa envolvendo seres humanos. Brasília; 2012 [cited 2019 Jul 22]; Available from: https://conselho.saude.gov.br/resolucoes/2012/Reso466.pdf

10. Nascimento AB. Supply and demand for health services in a sanitary territory as a contribution to the health care and health management. Rev. Gestão em Sist. Saúde [Internet]. 2016 [cited 2019 Jul 22]; 4(2):73-86. DOI: http://dx.doi.org/10.5585/rgss.v4i2.162

11. Campos RTO, Ferrer AL, Gama CAP, Campos GWS, Trapé TL, Dantas DV. Avaliação da qualidade do acesso na atenção primária de uma grande cidade brasileira na perspectiva dos usuarios. Saúde debate [Internet]. 2014 [cited 2019 Jul 22]; 38(spec):25264. DOI: http://dx.doi.org/10.5935/0103-1104.2014S019

12. Asensi FD. Judicialization, right to health and prevention. RECIIS - Rev. Eletron Comun Inf. Inov. Saúde [Internet]. 2015 [cited 2019 Jul 22]; 9(1):1-10. Available from: https://www.reciis.icict.fiocruz.br/index.php/reciis/article/view/935/1580

13. Santos D de S, Mishima SM, Merhy EE. Work process in Family Health Program: the potential of subjectivity of care for reconfiguration of the care model. Cien. Saude Colet. [Internet]. 2018 [cited 2020 Jul 22]; 23(3):861-70. DOI: http://dx.doi.org/10.1590/1413-81232018233.03102016

14. Costa AB, Gouvea PB, Cássia R De, Rangel T, Scnheider P, Alves F, et al. Nurses' perception on the reception and classification of risk in Primary Health Care. Enferm. actual Costa Rica [Internet]. 2018 [cited 2020 Jul 22]; 18(35):13. DOI: https://doi.org/10.15517/revenf.v0i35.32113

15. Santos AL, Rigotto RM. Territory and territorialization: incorporating the production, labor, environment, and health relations in primary health care. Trab. Educ. e Saúde [Internet]. 2010 [cited 2019 Jul 22]; 8(3):387-406. DOI: http://dx.doi.org/10.1590/S1981-77462010000300003

16. Lacerda e Silva T, Dias EC, Pessoa VM, Fernandes L da MM, Gomes EM. Occupational health in primary care: perceptions and practices in family health teams. Interface comun. saúde educ. [Internet]. 2014 [cited 2020 Jul 22]; 18(49):273-87. DOI: http://dx.doi.org/10.1590/1807-57622013.0227

17. Silva A, Ferraz L, Rodrigues-Junior SA. Worker's health actions developed by Primary Health Care in Chapecó, Santa Catarina, Brazil. Rev. Bras. Saúde Ocup. [Internet]. 2016 [cited 2019 Jul 22]; 41:e16. DOI: http://dx.doi.org/10.1590/2317-6369000122415

18. Cassettari SDSR, De Mello ALSF. Demand and type of care provided in emergency services in the city of Florianópolis, Brazil. Texto Contexto enferm. [Internet]. 2017 [cited 2019 Jul 22]; 26(1):e3400015. DOI: http://dx.doi.org/10.1590/010407072017003400015

19. Godoi VCG, Ganassin GS, Inoue KC, Gil NL de M. Reception with risk classification: characteristics of the Demand in an emergency unit. Cogitare Enferm. [Internet]. 2016 [cited 2019 Jul 22]; 21(3):1-8. DOI: http://dx.doi.org/10.5380/ce.v21i3.44664

20. Viacava F, Oliveira RAD de, Carvalho C de C, Laguardia J, Bellido JG. SUS: supply, access to and use of health services over the last 30 years. Cien. Saude Colet. [Internet]. 2018 [cited 2019 Jul 22]; 23(6):1751-62. DOI: http://dx.doi.org/10.1590/141381232018236.06022018

21. Araujo IMM de, Nascimento DF do, Pereira AM. Health regulation in the private sector: The case of ANS in Brazil and ERS in Portugal. Physis Rev. Saúde Coletiva [Internet]. 2018 [cited 2019 Jul 22]; 28(4):e280416. DOI: https://doi.org/10.1590/S0103-73312018280416

22. Facchini LA, Tomasi E, Dilélio AS. Quality of Primary Health Care in Brazil: advances, challenges and perspectives. Saúde Debate [Internet]. 2018 [cited 2019 Jul 22]; 42(spec):208-23. DOI: http://dx.doi.org/10.1590/0103-11042018s114

23. Paim JS. Thirty years of the Unified Health System (SUS). Ciênc saúde colet. [Internet]. 2018 [cited 2020 Jul 27]; 23(6). DOI: https://doi.org/10.1590/1413-81232018236.09172018

24. Sales OP, Vieira AFB, Martins AM, Garcia LG, Ferreira RKA. O Sistema Único de Saúde: desafios, avanços e debates em 30 anos de história. Rev. Humanidades e Inovação [Internet]. 2019 [cited 2020 Jul 27]; 6(17):55-65. Available from: https://revista.unitins.br/index.php/humanidadeseinovacao/article/view/1045

25. Silva CR, Carvalho BG, Cordoni Júnior L, Nunes E de FP de A. Difficulties in accessing services that are of medium complexity in small municipalities: a case study. Ciênc. Saúde Colet. [Internet]. 2017 [cited 2019 Jul 22]; 22(4):1109-20. DOI: http://dx.doi.org/10.1590/1413-81232017224.27002016

26. Inês D, Roloff T, Capa M, Almeida V, Bonow CA, Cezar-vaz R. Workplace health promotion actions in companies: an interdisciplinary contribution. Cogitare enferm. [Internet]. 2019 [cited 2019 Jul 22]; 24:e61042. DOI: http://dx.doi.org/10.5380/ce.v24i0.61042 